\title{
DRY TRANSFER BONDING OF POROUS SILICON MEMBRANES TO OSTE(+) POLYMER MICROFLUIDIC DEVICES
}

\author{
Farizah Saharil ${ }^{1}$, Kristinn B. Gylfason ${ }^{1, *}$, Yitong Liu ${ }^{1}$, Tommy Haraldsson ${ }^{1}$, \\ Paolo Bettotti ${ }^{2}$, Neeraj Kumar ${ }^{2}$, and Wouter van der Wijngaart ${ }^{1}$ \\ ${ }^{1}$ Microsystem Technology Lab, KTH Royal Institute of Technology, Stockholm, SWEDEN \\ ${ }^{2}$ Nanoscience Laboratory, Department of Physics, University of Trento, Povo (TN), ITALY
}

\begin{abstract}
We present low temperature transfer bonding of porous silicon membranes to polymeric microfluidic devices, using neither adhesives nor bond surface treatment. The transfer is enabled by a novel dual cure ternary monomer system containing thiolene and epoxy. The ability of the epoxy to react with almost any dry substrate provides a very versatile fabrication method. We characterize the two stage curing mechanism, describe the device fabrication, and evaluate the bond strength.
\end{abstract}

\section{INTRODUCTION}

The incorporation of membranes into microfluidics is of great interest for on-chip separation, gas-liquid contacting, and emulsification. Furthermore, membranes provide a large internal surface area for catalysis and adsorption. Among membrane materials, porous silicon has shown great potential for microfluidic applications, including biosensing [1] and filtering [2], due to its tunable bulk nanostructure that can be obtained from standard silicon wafers by low cost wafer-scale electrochemical etching. The most popular methods for incorporating membranes in microfluidics are gluing and clamping [3]. However, both methods have serious disadvantages: during manufacturing, liquid glue is sucked into the membrane, blocking its function, whereas during operation, capillary forces suck liquid between imperfectly sealed clamped layers. Dry bonding the membranes directly to the microfluidic device elegantly solves these problems, but no simple and generic such method was previously available $[4,5]$.

We recently introduced a family of off-stoichiometry thiol-ene (OSTE) polymers, specifically developed to replace PDMS in microfluidics [6]. OSTE can be dry bonded to pre-functionalized substrates [7] or to gold [8].

Here, we introduce a novel dual cure offstoichiometry-thiol-ene-epoxy $(\mathrm{OSTE}(+))$ polymer material, as an extension to the OSTE family. By adding epoxy to the OSTE polymer, it gains the ability to directly react with almost any dry surface. By utilizing OSTE $(+)$, we demonstrate dry transfer bonding of porous silicon membranes from their source wafer directly to a polymeric chip. The transfer is enabled by the unique dual cure process of OSTE $(+)$ : after the first stage UV cure of the prepolymer, and its subsequent demolding, the OSTE $(+)$ features a compliant surface containing epoxy and thiol groups for bonding. While after the second stage thermal cure of the bonded structure, the OSTE $(+)$ hardens to provide the mechanical stiffness, stability, and chemical inertness, needed for microfluidic applications. The novel and unique features of the method include: i) not requiring adhesives; ii) not requiring additional surface treatment; hence being iii) fast; and iv) simple.
The method enables the integration of nano-engineered porous silicon in cost-sensitive applications, such as nextgeneration single-use in-vitro diagnostic tests.

\section{OSTE(+) MATERIAL PROPERTIES}

The novel OSTE $(+)$ polymer formulation consists of thiol (Pentaerythritol tetrakis (2-mercaptoacetate)), allyl (triallyl-1,3,5-triazine-2,4,6(1H,3H,5H)-trione) and epoxy (bisphenol A diglycidyl ether) monomers (all acquired from Sigma-Aldrich). The stoichiometric ratios of the OSTE $(+)$ used here were 1.5/1.0/0.5, for thiol, allyl, and epoxy, respectively.

In the dual cure process, the thiol and allyl react at room temperature in the first step, via an alternating radical copolymerization initiated by Lucirin TPO (BASF) and UV-light (Fig. 1 a). We use a medium pressure mercury lamp equipped with a $365 \mathrm{~nm}$ band-pass filter at $6 \mathrm{~mW} / \mathrm{cm}^{2}$ for $10 \mathrm{~s}$. In this step, all allyl and half the thiols are consumed, resulting in a partially polymerized soft material. This intermediate product is ideal for direct dry bonding to many kinds of substrates, such as silicon and glass, since it is compliant to irregularities on the substrate, and is able to form covalent epoxy and thiol links upon further cure. The full consumption of allyl is verified in the Raman spectra of the allyl peak at $1645 \mathrm{~cm}^{-1}$, shown in Fig. 2 a. In contrast, the Raman spectra of the thiol peak at $2575 \mathrm{~cm}^{-1}$ in Fig. $2 \mathrm{~b}$ confirms the thiol residue after the first stage cure. No suitable peak is available to monitor the epoxy.

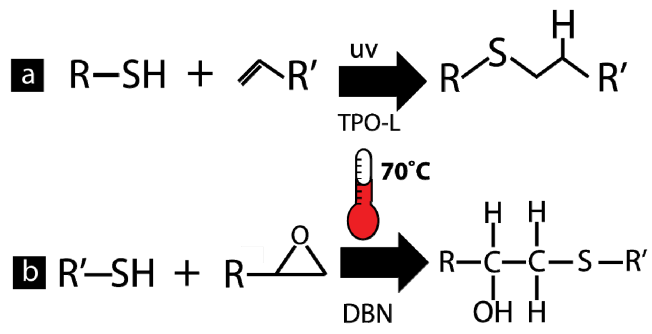

Figure 1: The reaction mechanism for the dual cure ternary monomer system, OSTE(+). a) In the first stage $U V$ cure, half the thiol reacts with the allyl, initiated by $T P O-L . b)$ In the second stage cure, the remaining thiol reacts with epoxy, initiated by $D B N$ at $70^{\circ} \mathrm{C}$.

In the second cure, the thiol and epoxy react, via an alternating anionic mechanism initiated by DBN $(1,5-$ diazabicyclo[4.3.0]non-5-ene, Sigma-Aldrich) and temperature $\left(70^{\circ} \mathrm{C}, 2 \mathrm{~h}\right) \quad$ (Fig. $\left.1 \mathrm{~b}\right)$. The second cure affords covalent bonding to hard substrates and yields materials with glass transitions temperature $\left(\mathrm{T}_{\mathrm{g}}\right)$ above $72^{\circ} \mathrm{C}$. As seen in the Raman spectra of the thiol peak in Fig. 2 b, the thiol is fully consumed in the second cure. 

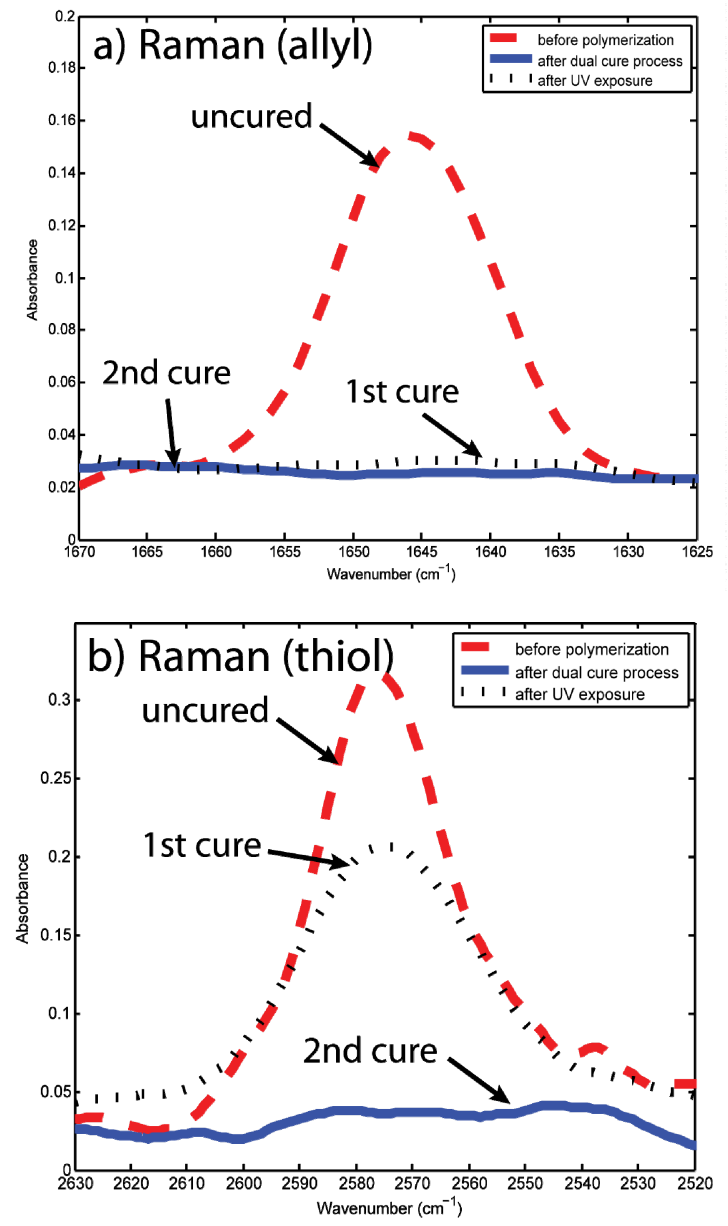

Figure 2: Raman spectra of: a) the allyl peak and b) the thiol peak, at each stage of the polymerization.

\section{DEVICE FABRICATION}

Demonstrator devices consist of $15 \times 15 \times 1 \mathrm{~mm}^{3}$ microfluidic OSTE $(+)$ chips, onto which $6 \mathrm{~mm}$ diameter, $30 \mu \mathrm{m}$ thick porous silicon membranes (50\% porosity, 70 $\mathrm{nm}$ pore size) are bonded. Fig. 3 shows a scanning electron image (SEM) of the porous membrane before bonding. The full device fabrication sequence is shown in Fig. 4. First, the porous silicon layer is formed by electrochemical etching and a final current pulse is applied to partially release the membrane from the silicon substrate [9] (Fig. 4 a).

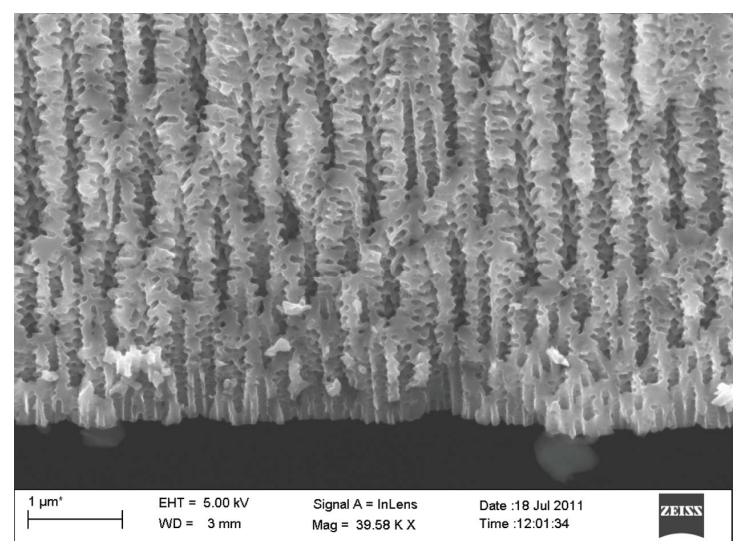

Figure 3: An SEM picture showing a cross-section of the porous silicon membrane before the transfer bonding.
The liquid OSTE $(+)$ prepolymer is cast in a PDMS mold, and the first curing step initiated by UV (Fig. 4 b). At this stage, the OSTE(+) is soft and the surface sticky. The OSTE $(+)$ is then released from the mold, and through holes are drilled prior to the transfer bonding. The porous membrane is thereafter transfer bonded to the $\operatorname{OSTE}(+)$ chip (Fig. $4 \mathrm{c}$ ). Finally, the chips are cured at $70^{\circ} \mathrm{C}$ for 2 hours to complete the polymerization. Fig. 5 shows the completed devices.

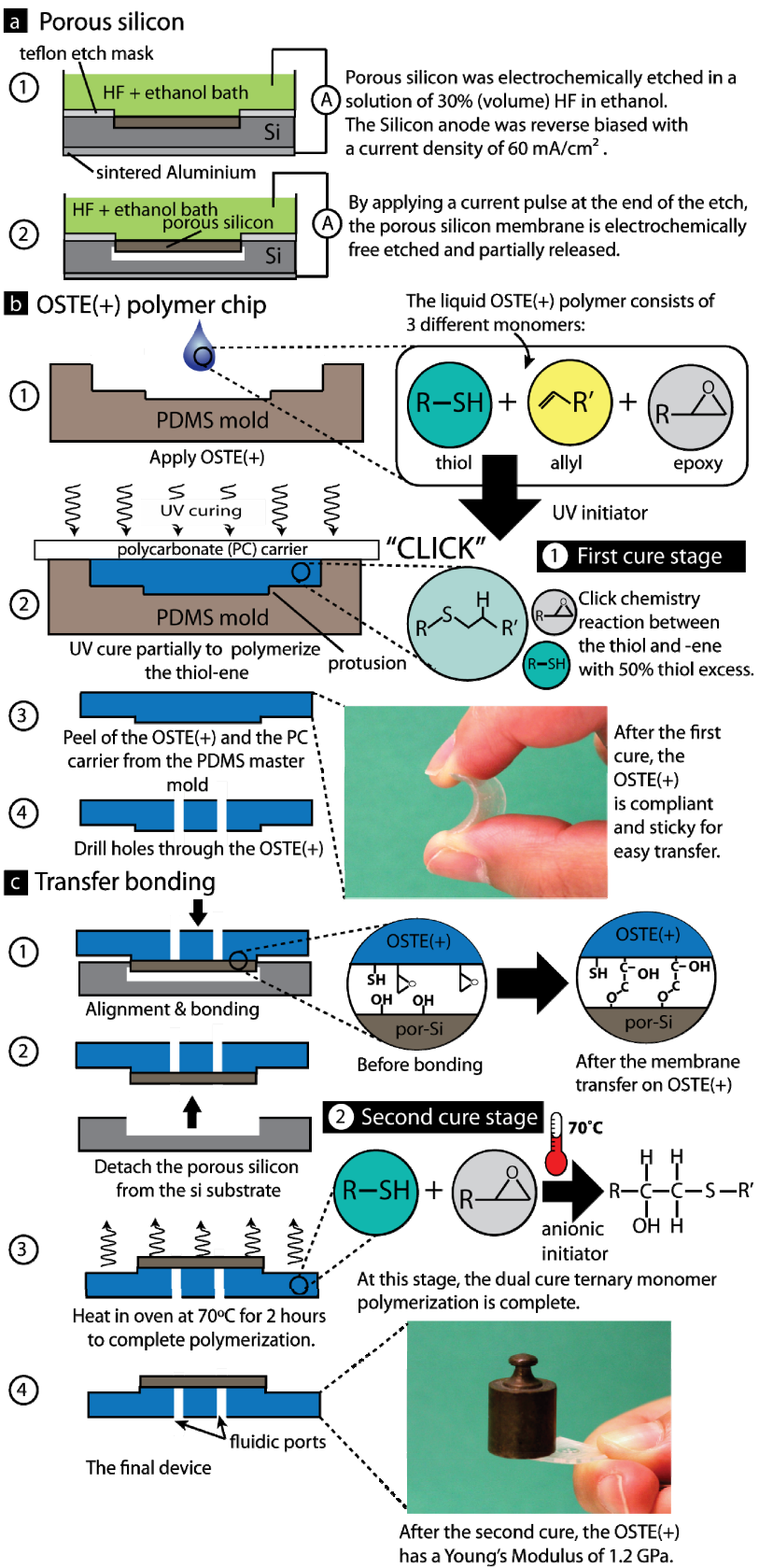

Figure 4: a) The porous silicon etching. b) Casting of the OSTE(+) chip, by the first curing step. c) Dry transfer bonding of the porous silicon membrane onto the OSTE (+) polymer chip and second stage cure. 


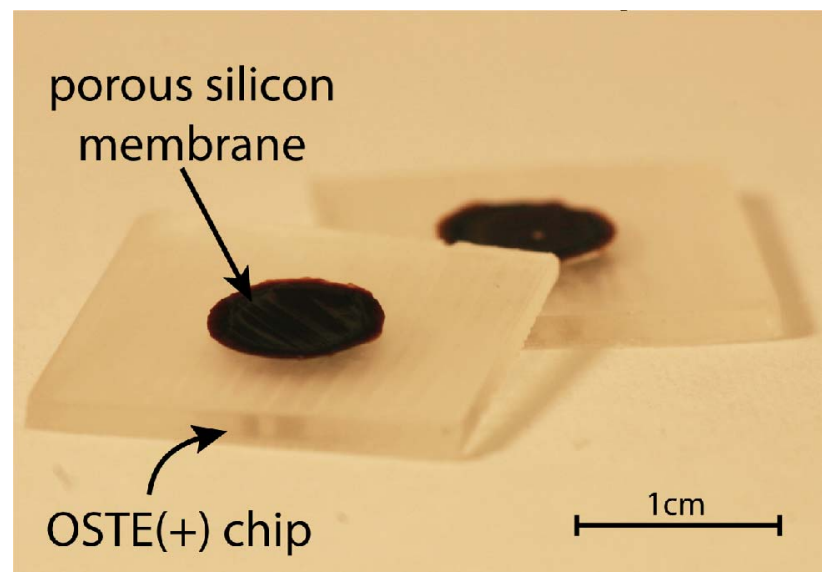

Figure 5: A photograph of the transferred porous silicon membranes onto OSTE(+) polymer chips.

\section{DEVICE CHARACTERIZATION}

First, the final OSTE(+) material properties were measured. A $\mathrm{T}_{\mathrm{g}}$ of $72^{\circ} \mathrm{C}$ and a Young's modulus of $1.2 \mathrm{GPa}$ were measured using dynamic mechanical analysis, showing the suitability of the $\operatorname{OSTE}(+)$ material as a porous silicon membrane carrier. The porous silicon contact angle can be modified after assembly: membranes are hydrophobic after the electro-chemical etching (Fig. 6 a), but become very hydrophilic (spreading wetting) after $\mathrm{O}_{2}$ plasma treatment of the completed chips (Fig. 6 b)

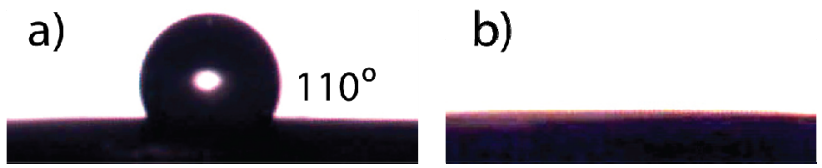

Figure 6: a) The contact angle of the porous silicon before the $\mathrm{O}_{2}$ plasma treatment. b) After the plasma treatment, the membrane is very hydrophilic and spreading wets.

For bond strength characterization, the chips were mounted in a flow cell. When increasing pressure was applied at the fluidic through holes, the membranes cracked before any leakage was observed at the bond interface, demonstrating the good sealing between the OSTE $(+)$ and the porous membrane (Fig 7 a). Finally we tested the use of the porSi device for filtering. DI water containing $10 \mu \mathrm{m}$ fluorescent beads was flown via the fluidic ports through the membrane. Fig. $7 \mathrm{~b}$ shows the capture of the beads on the porSi surface.
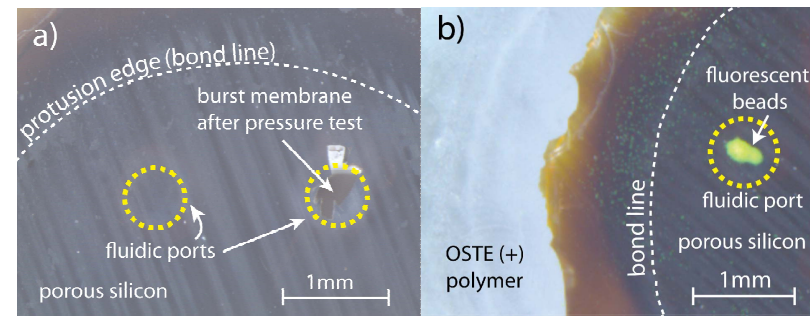

Figure 7: a) When pressure is applied to the OSTE(+) chip, the membrane bursts before the bonded interface leaks. b) Successful filtering of $10 \mu \mathrm{m}$ fluorescent beads.

\section{CONCLUSIONS}

We have demonstrated a simple and fast way to transfer porous silicon membranes to polymeric microfluidic devices, using neither adhesives nor bond surface treatment. The transfer is enabled by the development of a new polymer formulation, $\operatorname{OSTE}(+)$, which features a dual cure polymerization specifically designed for bonding. We have characterized the two stage curing mechanism by Raman spectroscopy. To illustrate the usefulness of the method, we fabricated a polymeric microfluidic device with an integrated porous silicon membrane. We evaluated the membrane bond strength, showed that its wetting properties have by modified, and demonstrated its use for filtering.

\section{ACKNOWLEDGEMENTS}

This work was partly financed by the European Commission through the project FP7-257401 POSITIVE.

\section{REFERENCES}

[1] V. Mulloni and L. Pavesi, "Porous silicon microcavities as optical chemical sensors," Appl. Phys. Lett., vol. 76, pp. 2523-2525, 2000.

[2] R. W. Tjerkstra, et al., "Multi-walled microchannels: free-standing porous silicon membranes for use in TAS," J. Microelectromech. Systems, vol. 9, pp. 495-501, Dec. 2000.

[3] J. D. Jong, et al., "Membranes and microfluidics: a review," Lab on a Chip, vol. 6, pp. 1125-1139, 2006.

[4] M. A. Eddings, et al., "Determining the optimal PDMS-PDMS bonding technique for microfluidic devices," J. Micromechanics and Microengineering, vol. 18, no. 6, pp. $067001+, 2008$.

[5] L. Riegger, et al., "Adhesive bonding of microfluidic chips: influence of process parameters," J. Micromechanics and Microengineering, vol. 20, pp. $087003+$, Aug. 2010.

[6] C. F. Carlborg, et al., "Beyond PDMS: offstoichiometry thiol-ene (OSTE) based soft lithography for rapid prototyping of microfluidic devices," Lab on a Chip, vol. 11, pp. 3136-3147, 2011.

[7] C. F. Carlborg, et al., "Low temperature "click" wafer bonding of off-stoichiometry thiol-ene (OSTE) polymers to silicon," in Proceedings Micro Total Analysis Systems (microTAS), Oct. 2011.

[8] N. Sandström, et al., "One step integration of gold coated sensors in OSTE polymer cartridges by low temperature dry bonding," in Proceedings IEEE International Conference on Solid-State Sensors, Actuators, and Microsystems (Transducers), Jun. 2011, pp. 2778-2781.

[9] M. Ghulinyan, et al., "Free-standing porous silicon single and multiple optical cavities," J. Appl. Phys., vol. 93, pp. 9724-9729, 2003.

\section{CONTACT}

*K.B. Gylfason, tel: +46 879092 31, fax: +4681008 58; kristinn.gylfason@ee.kth.se 